Check for updates

Cite this: RSC Adv., 2019, 9, 34065

\title{
Selective oxidation of methacrolein to methacrylic acid over $\mathrm{H}_{4} \mathrm{PMO}_{11} \mathrm{VO}_{40} / \mathrm{C}_{3} \mathrm{~N}_{4}-\mathrm{SBA}-15 \dagger$
}

\author{
Lilong Zhou, (D) a Shanshan Zhang, ${ }^{a}$ Zhengjie Li, ${ }^{a}$ Jason Scott, (D) ${ }^{\mathrm{b}}$ Zhikun Zhang, ${ }^{\mathrm{a}}$ \\ Runjing Liu*a and Jimmy Yun*ab
}

Molybdovanadylphosphoric acid (HPMV) was supported on a carbon nitride-modified SBA-15 (CN-SBA-15) molecular sieve to enhance its catalytic performance for oxidation of methacrolein (MAL) to methacrylic acid (MAA). HPMV/CN-SBA exhibited increased catalytic activity (20\%) and five times greater MAA selectivity (98.9\%) compared to bulk HPMV. HPMV supported on CN-SBA-15 exhibited much better catalytic performance as compared to that on other supports, such as KIT-6, HY zeolite, $\mathrm{TiO}_{2}, \mathrm{Al}_{2} \mathrm{O}_{3}$, $\mathrm{SiO}_{2}, \mathrm{CNTS}$, and $\mathrm{NH}_{3}$-modified CNTs. The supported HPMV was well characterized by FT-IR, XRD, SEM, $\mathrm{N}_{2}$ physical desorption, TG-DTA, $\mathrm{NH}_{3}-\mathrm{TPD}, \mathrm{CO}_{2}-\mathrm{TPD}, \mathrm{XPS}$, and solid-state NMR. The CN minimized the interaction between the silica support and HPMV. HPMV was successfully separated from SBA-15, which was restricted by $\mathrm{CN}$ to increase stability and prevent interaction between the catalysts and support that would lead to decomposition of the catalysts during calcination and reaction. HPMV reacted with amino groups on the $\mathrm{CN}$, which improved MAA selectivity and enhanced the thermal stability of the supported heteropoly acid (HPA) catalysts. This work identifies a new approach to preparing highly efficient and stable supported HPA catalysts for oxidation reactions.

Received 3rd August 2019

Accepted 2nd October 2019

DOI: 10.1039/c9ra06021a

rsc.li/rsc-advances these, silica is considered a good option due to its thermal stability under high-temperature reaction conditions in conjunction with its low cost. Legagneux et al. supported $\mathrm{H}_{4} \mathrm{SiW}_{12} \mathrm{O}_{40}$ and $\mathrm{H}_{3} \mathrm{PW}_{12} \mathrm{O}_{40}$ on porous $\mathrm{SiO}_{2}$ using maceration, and both HPAs reacted with the silica support to give [= $\left.\mathrm{SiOH}_{2}\right]_{x} \mathrm{H}_{4-x} \mathrm{SiW}_{12} \mathrm{O}_{40}$ and $\left[=\mathrm{SiOH}_{2}\right]_{3} \mathrm{PW}_{12} \mathrm{O}_{40}$, respectively, which led to partial decomposition of the Keggin structure upon heating. ${ }^{9}$ Kanno et al. supported $\mathrm{HPMV}$ on $\mathrm{SiO}_{2}$ for oxidation of MAL to MAA; however, the supported HPAV decomposed to $\mathrm{MoO}_{3}$ during the reaction. ${ }^{\mathbf{1 1}}$ To surmount the decomposition problem, they instead loaded HPMV onto $\mathrm{NH}_{3}$-modified $\mathrm{SiO}_{2}$. The modified catalyst exhibited better catalytic performance with MAL conversion and MAA selectivity at $9 \%$ and $89 \%$, respectively. ${ }^{12}$ However, the $\mathrm{NH}_{3}$-modification of $\mathrm{SiO}_{2}$ was performed at $900{ }^{\circ} \mathrm{C}$, which is not suitable for scaling up of the reaction. Despite the improved performance, the supported catalysts continued to display poor thermal stability under the reaction conditions, and MAL conversion remained too low. It has been reported that the substandard thermal stability originates from the reaction between the $\mathrm{H}_{4} \mathrm{PMo}_{11} \mathrm{VO}_{40}$ catalyst and the silica support, whereby silicon atoms replace the central atom of the HPA structure. ${ }^{17}$

Consequently, modifications to the silica surface so as to enhance thermal stability and improve the catalytic performance of the supported HPA catalyst are still required. One potential support modifier is carbon nitride $(\mathrm{CN})$. Incorporating nitrogen atoms into a carbon nanostructure has been shown to enhance the conductive, mechanical, field-emission, and
${ }^{a}$ College of Chemical and Pharmaceutical Engineering, Hebei University of Science and Technology, Shijiazhuang, Hebei province, 050018, P. R. China.E-mail: liurj2002@ 163.com

${ }^{b}$ School of Chemical Engineering, The University of New South Wales, Sydney, NSW 2052, Australia.E-mail: jimmy.yun@unsw.edu.au

$\dagger$ Electronic supplementary information (ESI) available. See DOI: 10.1039/c9ra06021a 
energy-storage properties of carbon materials. ${ }^{18-31}$ More importantly, the carbon nitride surface possesses high levels of amino groups, ${ }^{22}$ and adding $\mathrm{NH}_{3}$ to heteropolyacid catalysts has been previously reported to improve its performance for selective oxidation reactions. ${ }^{32}$ The potential then exists to use $\mathrm{CN}$ as a barrier to HPA degradation when it is supported on silica while concurrently promoting HPA catalytic performance via $\mathrm{NH}_{3}$ inclusion into the structure.

In the work presented here, the prospect of using $\mathrm{CN}$ as a favourable modifier for silica-supported HPA catalysts was examined. HPMV was loaded onto a CN-modified SBA-15 support and assessed as a catalyst for oxidizing MAL to MAA. The CN loading, synthesis conditions, and preparation time of the catalysts were optimized.

\section{Results and discussion}

\section{Catalyst characteristics}

FTIR. FTIR spectra of neat SBA-15, neat CN, CN-SBA, neat calcined HPAV, and 2HPMV/CN-SBA are shown in Fig. 1. The typical vibrational bands for silica are at 3436, 1086, and $807.6 \mathrm{~cm}^{-1}$, which can be assigned to $v s .(\mathrm{O}-\mathrm{H}), v s$. ( $\left.\mathrm{Si}-\mathrm{O}-\mathrm{Si}\right)$, and vs.(Si-O), respectively. ${ }^{35} \mathrm{~A}$ series of vibrational bands seen at 1237-1578 $\mathrm{cm}^{-1}$ correspond to aromatic $\mathrm{C}-\mathrm{N}$ and aromatic carbon rings. ${ }^{36}$ The vibrational band of $\mathrm{N}-\mathrm{H}$ in amino groups is shown at $3259 \mathrm{~cm}^{-1},{ }^{36}$ suggesting the existence of amino groups in $\mathrm{C}_{3} \mathrm{~N}_{4}$. The composite CN-SBA spectrum exhibits proponents of both the neat SBA-15 and neat CN spectra, indicating that $\mathrm{C}_{3} \mathrm{~N}_{4}$ was successfully loaded onto the silica support. Four vibrational bands occur at 1065, 960, 865, and $802 \mathrm{~cm}^{-1}$ in the spectrum of HPMV, which can be assigned to $v s .(\mathrm{P}-\mathrm{O}), v s .(\mathrm{Mo}=$ $\left.\mathrm{O}_{\mathrm{d}}\right)$, vs. $\left(\mathrm{Mo}-\mathrm{O}_{\mathrm{b}}-\mathrm{Mo}\right)$, and $v s .\left(\mathrm{Mo}-\mathrm{O}_{\mathrm{c}}-\mathrm{Mo}\right)$, respectively, and these are the characteristic vibration bands of the Keggin structure. ${ }^{34}$ The vibrational band at $1036 \mathrm{~cm}^{-1}$ can be assigned to $v s$. $(\mathrm{V}-\mathrm{O})$ in $\mathrm{VO}^{2+}$, which was formed from the migration of $\mathrm{V}$ atoms in the Keggin structure to the secondary structure during calcinations. ${ }^{37}$

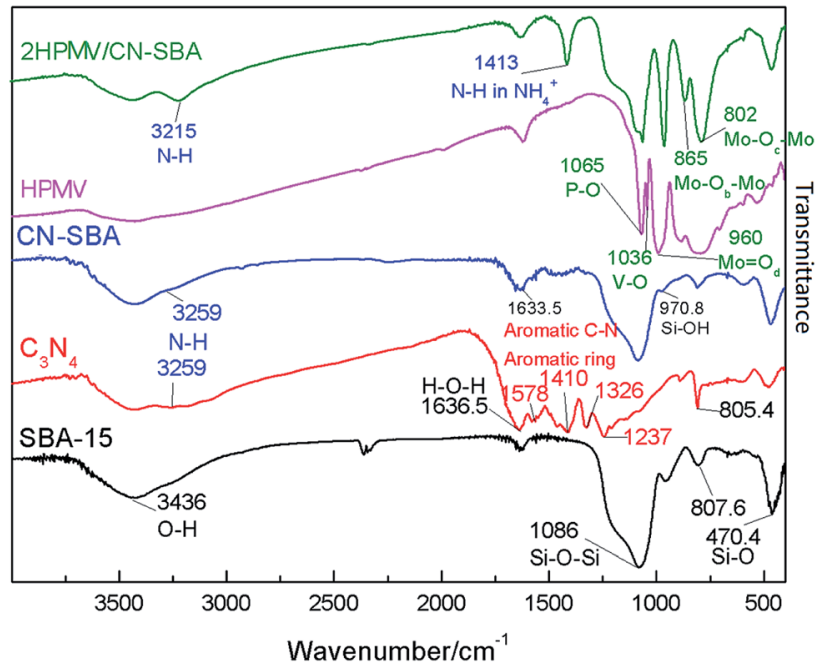

Fig. 1 FT-IR spectra of SBA-15, CN, CN-SBA, calcined HPMV, and 2HPMV/CN-SBA.
The four vibrational bands that are characteristic of a Keggin structure also emerged in the 2HPMV/CN-SBA spectrum. When compared with neat HPMV (Fig. S1 $\dagger$ ), the $v s .(\mathrm{Mo}=\mathrm{Od})$ of $x \mathrm{HPMV} / \mathrm{CN}-\mathrm{SBA}$ shows a shift from 961 to $960 \mathrm{~cm}^{-1}$, vs. (Mo-O $\mathrm{b}_{\mathrm{b}}-\mathrm{Mo}$ ) has shifted from 874 to $865 \mathrm{~cm}^{-1}$, and $v s .\left(\mathrm{Mo}-\mathrm{O}_{\mathrm{c}}-\mathrm{Mo}\right)$ has shifted from 780 to $801.9 \mathrm{~cm}^{-1}$, while $v s .(\mathrm{P}-\mathrm{O})$ remains unchanged. The shifts suggest that there has been an interaction between the HPAV and amino groups on the CN-SBA support, and it also suggests that HPMV was successfully supported on CN-SBA. A new vibrational band emerged at $1413 \mathrm{~cm}^{-1}$, which can be assigned to vs. $(\mathrm{N}-\mathrm{H})$ in $\mathrm{NH}_{4}{ }^{+} \cdot{ }^{39}$ The band indicates that HPMV reacted with the amino groups on the CN-SBA surface to form HPA ammonium salt.

Fig. S5-S9† shows the FTIR spectra of the HPMV, noncalcined catalysts, calcined catalysts, and used catalysts with different HPMV loadings, as well as catalysts prepared at different temperatures with different synthesis time. Apart from the $\mathrm{V}-\mathrm{O}$ vibrational bands in $\mathrm{VO}^{2+}$ appearing in the calcined and used HPMV spectra, there is no obvious difference between the calcined HPMV spectrum and the noncalcined HPMV or used HPMV spectra (Fig. S1 $\dagger$ ). The spectra of the as-prepared HPMV/CN-SBA exhibited characteristic Keggin structure $\left(1060,960,870\right.$, and $\left.796 \mathrm{~cm}^{-1}\right)$, silica (1086 and $465 \mathrm{~cm}^{-1}$ ), and CN (1460 and $1412 \mathrm{~cm}^{-1}$ ) vibrational bands, which also indicated that HPMV was successfully loaded onto CN-SBA (Fig. 1, S5-S9†).

After calcination, a new band emerged at $1036 \mathrm{~cm}^{-1}$ that was assigned to the $\mathrm{V}-\mathrm{O}$ vibration band. Disappearance of the band at $1460 \mathrm{~cm}^{-1}$ may have occurred from the reaction between HPMV and amino groups during calcinations (Fig. S6 $\dagger$ ). After increasing the HPMV loading to $n=3$ (75 wt\%), the Mo-O vibrational band at $595 \mathrm{~cm}^{-1}$, belonging to $\mathrm{MoO}_{3}$, appears in the calcined and used catalyst spectra (Fig. S5-S7†). Appearance of the Mo-O vibration suggests that if excessive HPMV loading occurs, catalyst decomposition may be promoted. The preparation temperature and synthesis time have little influence on the structure of the supported catalysts (Fig. S8 and S9†) over the range considered.

X-ray diffraction. XRD patterns of SBA-15, CN, CN-SBA, HPMV, and 2HPMV/CN are provided in Fig. 2. Only a broad diffraction peak at $22.5^{\circ}$ was observed for the SBA-15 XRD patterns, which indicated an amorphous silica presence. In the case of neat $\mathrm{CN}$, a strong diffraction is present at $27^{\circ}$, which depicts a $0.323 \mathrm{~nm}$ lattice spacing and can be attributed to the interlayer distance between the carbon nitride sheets (i.e., resembling the 002 reflection of graphite). ${ }^{19} \mathrm{~A}$ broad diffraction peak at $12^{\circ}$ can be ascribed to the intralayer periodicity in $\mathrm{C}_{3} \mathrm{~N}_{4}$. The two reflections also emerge in the $\mathrm{CN}$ SBA pattern, illustrating the successful modification of SBA15 by $\mathrm{C}_{3} \mathrm{~N}_{4}$.

Typical HPA patterns with a Keggin structure are a feature of the neat HPMV sample, which can be associated with a triclinic crystal phase. ${ }^{32}$ When HPMV was loaded on CN-SBA, two HPMV crystal forms are evident in the $2 \mathrm{HPMV} / \mathrm{CN}-\mathrm{SBA}$ patterns, cubic and triclinic. The overlaying crystal spectra potentially reflect $\left(\mathrm{NH}_{4}\right)_{x} \mathrm{H}_{4-x} \mathrm{PMo}_{11} \mathrm{VO}_{40}$ (cubic crystal, $2 \theta=10.6^{\circ}, 15.0^{\circ}, 19.3^{\circ}$, 


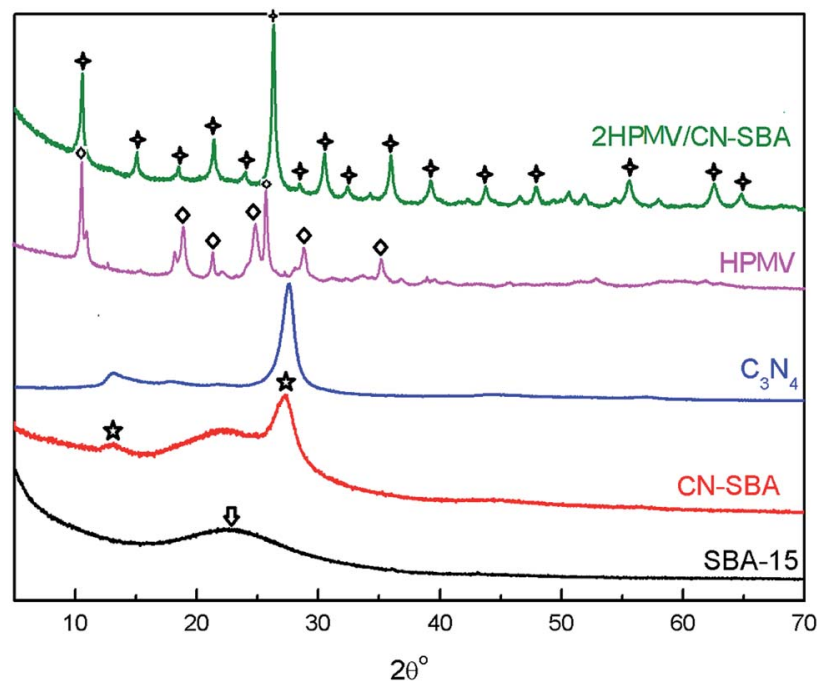

Fig. 2 XRD patterns of SBA-15, CN-SBA, $\mathrm{C}_{3} \mathrm{~N}_{4}$, calcined HPMV, and $2 \mathrm{HPMV} / \mathrm{CN}-\mathrm{SBA}$. Quadrangular star $=\left(\mathrm{NH}_{4}\right)_{x} \mathrm{H}_{4-x} \mathrm{PMO}_{11} \mathrm{VO}_{40} ;$ arrow $=$ amorphous silica; $\diamond=\mathrm{HPMV}$; $=\mathrm{C}_{3} \mathrm{~N}_{4}$.

$21.4^{\circ}, 26.3^{\circ}, 30.5^{\circ}$, and $36.0^{\circ}$ ) and $\mathrm{H}_{4} \mathrm{PMo}_{11} \mathrm{VO}_{40}$ (triclinic crystal, $2 \theta=9.0^{\circ}, 9.3^{\circ}, 18.5^{\circ}, 24.8^{\circ}$, and $28.7^{\circ}$ ) (Fig. S10†)..$^{32,39}$ The spectrum depicting $\left(\mathrm{NH}_{4}\right)_{x} \mathrm{H}_{4-x} \mathrm{PMO}_{11} \mathrm{VO}_{40}$ reveals that HPMV has reacted with amino groups on the CN-SBA surface. The diffraction patterns of $\mathrm{C}_{3} \mathrm{~N}_{4}$ also can be observed at $27^{\circ}$ for composite samples.

After calcining, the HPMV patterns have disappeared, with only $\left(\mathrm{NH}_{4}\right)_{x} \mathrm{H}_{4-x} \mathrm{PMo}_{11} \mathrm{VO}_{40}$ remaining. This suggests that HPMV continued to react with amino groups during calcination. However, when the HPMV loading was deficient (e.g., $0.5 \mathrm{HPMV} / \mathrm{CN}-\mathrm{SBA})$ or too high (4HPMV/CN-SBA, $5 \mathrm{HPMV} / \mathrm{CN}-$ SBA), diffraction patterns representing $\mathrm{MoO}_{3}$ and $\mathrm{V}_{2} \mathrm{O}_{5}\left(25.6^{\circ}\right.$ and $27.3^{\circ}, 20.5^{\circ}$, Fig. S10b $\dagger$ ) appeared for the calcined supported catalysts. The $\mathrm{MoO}_{3}$ and $\mathrm{V}_{2} \mathrm{O}_{5}$ originate from degradation of the supported HPA during calcination. $\mathrm{MoO}_{3}$ diffraction patterns are also observed in the used 3HPMV/CN-SBA, 4HPMV/ CN-SBA, and 5HPMV/CN-SBA patterns (Fig. S11†), which were generated from the decomposition of HPMV. It appears that if there is insufficient or excessive HPMV loaded onto the CN-SBA support, the thermal stability of HPA is diminished, and it undergoes partial decomposition. $\mathrm{MoO}_{3}$ diffraction patterns are also observed in the patterns of calcined 2HPMV/CN-SBA prepared at temperatures of $100{ }^{\circ} \mathrm{C}$ or greater and preparation times of $8 \mathrm{~h}$ or longer (Fig. S12 and S13†). The crystal diameters (Table S1 $\dagger$ ) calculated by the Scherrer formula show that the catalyst size decreased after being supported and increased with the increase in the loading amount. The findings indicate that an excessive preparation temperature and/or preparation time also lead to HPA decomposition.

Specific surface area and pore distribution. The $\mathrm{N}_{2}$ physical adsorption isotherms and pore diameter distributions for SBA-15, CN-SBA, and the supported catalysts are shown in Fig. S14, $\dagger$ with values provided in Table 1 . The isotherms reflect typical IV isotherms, indicating that all the materials are mesoporous. The neat SBA-15 surface area was $1018 \mathrm{~m}^{2}$ $\mathrm{g}^{-1}$ and decreased to $499.8 \mathrm{~m}^{2} \mathrm{~g}^{-1}$ upon modification with the $\mathrm{CN}$. There was a small increase in the average pore diameter (6.6 $\mathrm{nm}$ vs. $7.7 \mathrm{~nm}$ ), while the pore volume decreased following $\mathrm{C}_{3} \mathrm{~N}_{4}$ addition. The nature of the change in pore diameter and volume suggests that the decrease in surface area originated from partial blocking of smaller pores by $\mathrm{C}_{3} \mathrm{~N}_{4}$. When HPMV is loaded onto the CN-SBA, the surface area further decreases to $118 \mathrm{~m}^{2} \mathrm{~g}^{-1}$ (2HPMV/CN-SBA). With increased HPMV loading, the surface area of the supported catalysts decreased (Table 1). A new pore size with a diameter of $4 \mathrm{~nm}$ emerged following HPMV addition to the CN-SBA. The volume ratio of the smaller $(4 \mathrm{~nm})$ pores to the larger (approximately $7 \mathrm{~nm}$ ) pores increased with increasing HPMV (Fig. S15-S19†). These findings suggest that the supported HPMV enters the available pores in the CN-SBA, which results in a decrease in the surface area and a narrowing of pores. Relative to bulk HPMV and NHPMV, the surface area of HPMV/CN-SBA is considerably larger.

Catalyst morphology. As seen in Fig. 3, the microscopic morphology of CN-SBA is similar to that of SBA-15 in terms of the cylindrical SBA-15 assembled beams, which indicates that the CN does not impact the SBA-15 structure. When loaded with HPMV, the underlying morphology of the SBA-15 again remained unchanged, although some additional plate-shaped particles appeared on the support surface. With increasing HPMV loading, the amount and size of the plate-shaped particles increases (Fig. S20 $†$ ). At a HPMV loading of three and beyond, cubic particles with a diameter greater than $300 \mathrm{~nm}$ have appeared at the support corner. This

Table 1 The Brunauer-Emmett-Teller (BET) results of catalysts with different loading amounts

\begin{tabular}{lccc}
\hline Catalysts & Specific surface area $/ \mathrm{m}^{3} \mathrm{~g}^{-1}$ & Pore volume $/ \mathrm{cm}^{3} \mathrm{~g}^{-1}$ & Average pore diameter/nm \\
\hline HPMV & 3.3 & 0.014 & 17.8 \\
$\mathrm{NH}_{4}$ PMV & 2.4 & 0.008 & 15.6 \\
SBA-15 & 1018 & 1.307 & 6.6 \\
CN-SBA & 499.4 & 1.019 & 7.7 \\
$0.5 \mathrm{HPMV} /$ CN-SBA & 165.6 & 0.40 & 8.2 \\
1HPMV/CN-SBA & 175.2 & 0.32 & 7.8 \\
2HPMV/CN-SBA & 118.0 & 0.20 & 8.1 \\
3HPMV/CN-SBA & 66.7 & 0.11 & 7.3 \\
4HPMV/CN-SBA & 66.4 & 0.086 & 5.7 \\
5 HPMV/CN-SBA & 50.4 & 0.089 & 5.9
\end{tabular}




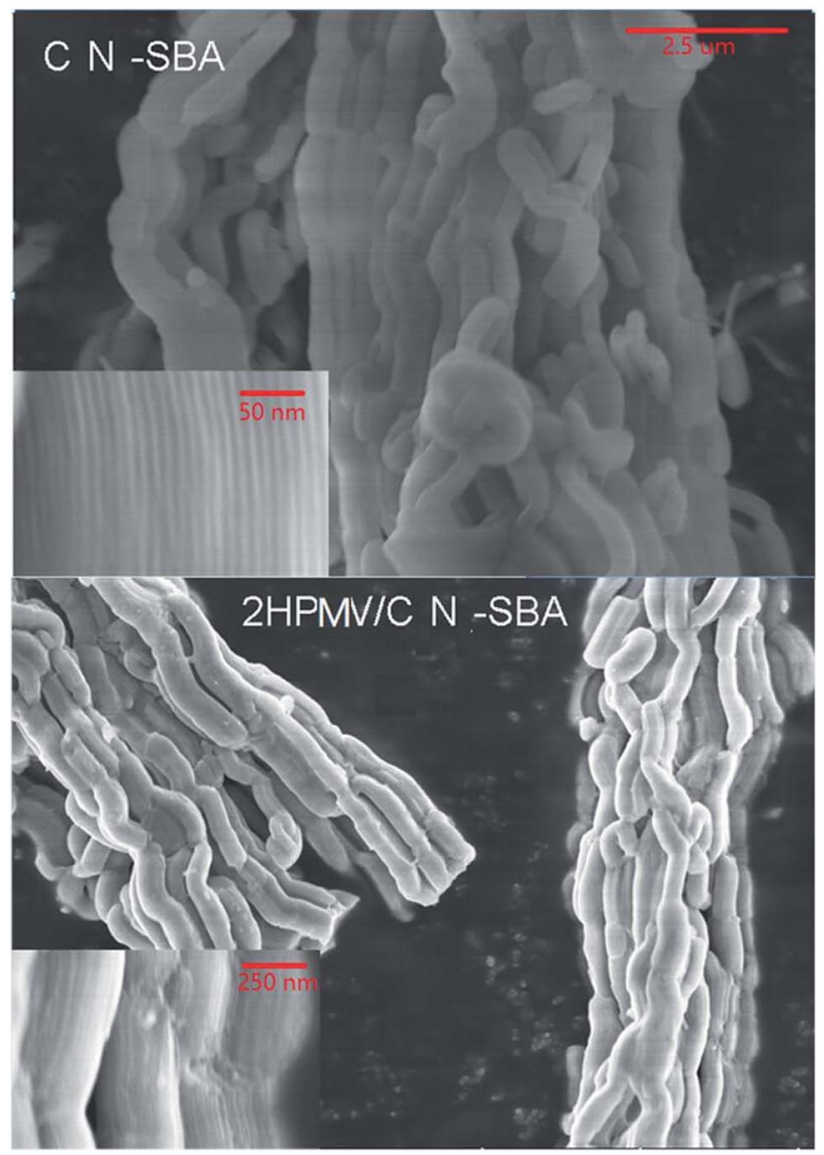

Fig. 3 SEM images of CN-SBA and 2HPMV/CN-SBA.

demonstrates that these plate-shaped and cubic particles were $\left(\mathrm{NH}_{4}\right)_{x} \mathrm{H}_{4-x} \mathrm{PMO}_{11} \mathrm{VO}_{40}$ crystallized on the outer surface of the support, as confirmed by XRD (Fig. S10†).

With increasing HPMV loading, the large plate per cubic particles firstly appear at the end, on the corners, and in the cracks of the support. The appearance of the plate per cubic originates from the HPMV initially entering the pores of the support and reacting with amino groups on the pore walls during preparation. With increasing HPMV loading, NHPMV crystals continued to accumulate in the pores, and eventually grew out of the pores to form large particles. Pore exits are absent at the end, corners, and cracks of the support, with the large particles consequently growing at these points. This may also account for the decrease in surface area with increased HPMV loading. Concurrently, HPMV may also react with amino groups on the outer surface of the support to form the plateshaped particles. The BET results and scanning electron microscopy (SEM) images indicate that excessive HPMV loading decreases the surface area and is detrimental for the pore structure of the support.

Thermal stability of the catalysts. Thermal stability is a key factor that has restricted the application of supported HPA catalysts. Thermogravimetry-differential thermal analysis (TGDTA) was performed to determine the thermal stability of the supported catalysts (Fig. 4 and S21†). In the case of carbon
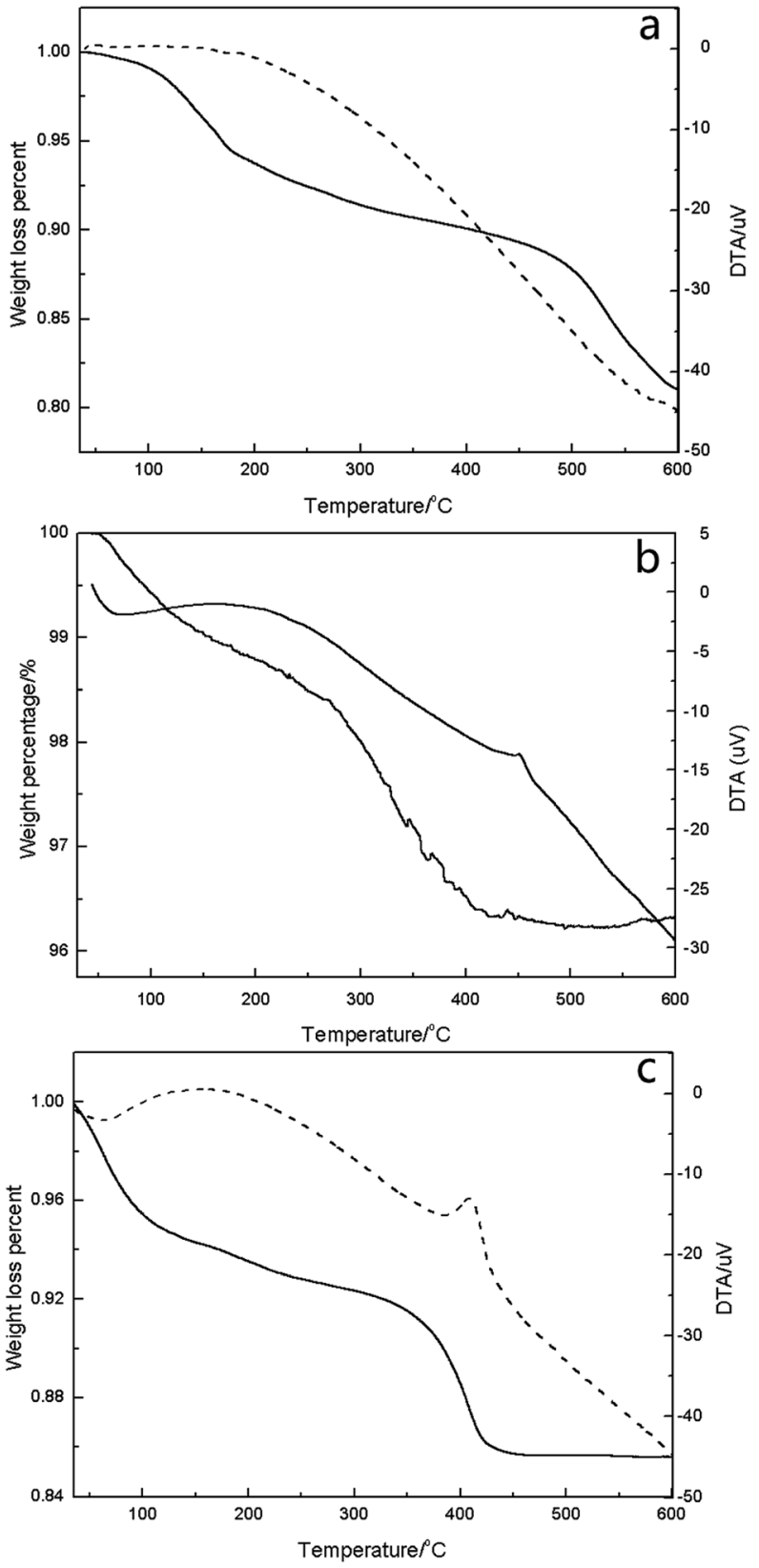

Fig. 4 TG-DTA curves of CN-SBA, HPMV and 2HPMV/CN-SBA ((a) CN-SBA; (b). HPMV; (c) $2 \mathrm{HPMV/CN-SBA).}$

nitride-modified SBA-15 (Fig. 5a), three weight loss stages are apparent upon heating in air. The first stage below $200{ }^{\circ} \mathrm{C}$ can be assigned to the desorption of adsorbed water. The weight loss between 200 and $500{ }^{\circ} \mathrm{C}$ can be attributed to ammonia desorption..$^{20}$ At a temperature higher than $500{ }^{\circ} \mathrm{C}$, the weight loss accounts for $\mathrm{CN}$ oxidation (by oxygen). ${ }^{24}$ As seen in Fig. $5 \mathrm{~b}$, the weight of HPMV decreased by approximately $1.2 \%$ at $200^{\circ} \mathrm{C}$, which can be assigned to the loss of adsorbed and crystalline water. ${ }^{33}$ By $460{ }^{\circ} \mathrm{C}$, the constitutional water was removed, resulting in Keggin-type compounds with oxygen vacancies, which finally decomposed to the oxides as the temperature reached approximately $640{ }^{\circ} \mathrm{C} .^{40,41}$ 

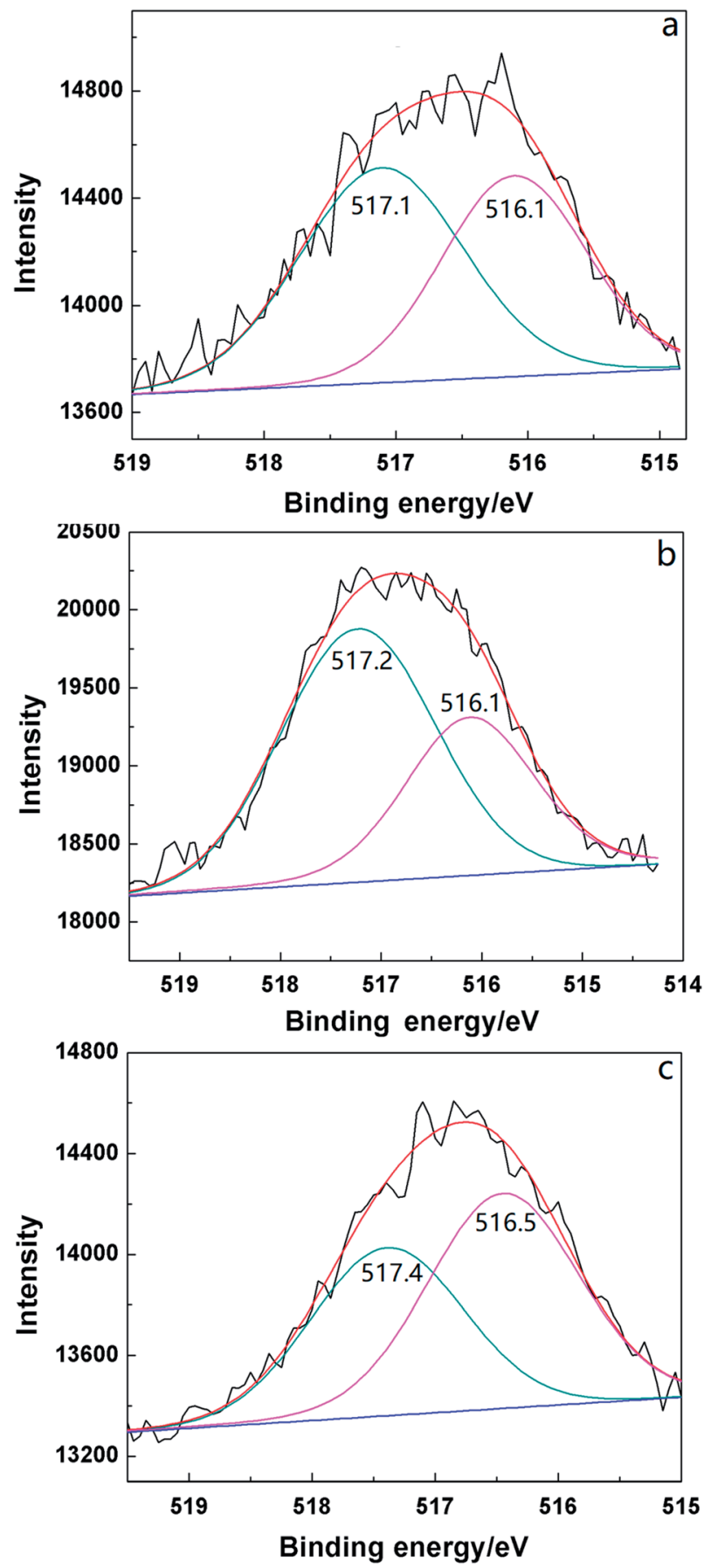

Fig. 5 XPS spectra of $V 2 p$ in 2 HPMV/CN-SBA (a) before and (b) after calcination, and (c) following the reaction.

In the case of $2 \mathrm{HPMV} / \mathrm{CN}-\mathrm{SBA}$ (Fig. $5 \mathrm{c}$ ), removal of the constitutional water had occurred at approximately $420{ }^{\circ} \mathrm{C}$, which was $30{ }^{\circ} \mathrm{C}$ lower than bulk HPMV. Subsequently, it appears that the thermal stability of HPA can be weakened when it is loaded on a support. The decrease of thermal stability of supported HPMV may be caused by the decrease in the catalyst crystal size. The reaction temperature for oxidizing MAL to MAA is 280 to $350{ }^{\circ} \mathrm{C},{ }^{10-13,17,32,34,37,39-43}$ and therefore, the thermal stability of HPMV/CN-SBA is sufficient for application in this reaction. The temperature decreases with increasing HPMV loading, at which the supported catalysts completely decomposed (Fig. S21 $\dagger$ ). It is apparent that an increased HPMV presence lowers the thermal stability of the supported catalyst.

Surface oxidation state. The XPS results are provided in Fig. 5, S22-S27 and Table S1. $\dagger$ As can be seen in Fig. S22, $\dagger$ only one peak is presented $(103.9 \mathrm{eV})$ in the ${ }^{29} \mathrm{Si} 2 \mathrm{p}$ spectrum of $\mathrm{CN}$ SBA, which can be assigned to the silicon species in SBA-15. ${ }^{38}$ Upon loading HPMV onto the support (Fig. S22 $\dagger$ ), the Si binding energy shifted to $103.3 \mathrm{eV}$ due to the interaction between HPMV and the support. Calcination did not alter the Si binding energy, although a further decrease (to $103.1 \mathrm{eV}$ ) was observed after the reaction. No other silicon species were detected in the three samples, which suggests that the interaction between HPMV and $\mathrm{C}_{3} \mathrm{~N}_{4}$ also caused little change in SBA- 15 .

There were at least four different nitride species present within the carbon nitride (Fig. S24 $\dagger$ ). The peak at $400.6 \mathrm{eV}$ can be attributed to amino groups and is analogous to nitride species on carbon nitride. ${ }^{24}$ The other three peaks at 400.0 , 399.4 , and $398.6 \mathrm{eV}$ can be assigned to nitrogen species in the form of $\mathrm{N}-\mathrm{O}, \mathrm{C}-\mathrm{N}$, and $\mathrm{N}-\mathrm{H}$, respectively. ${ }^{25,27,28}$ These findings confirm the existence of carbon nitride and amino groups on the support surface.

Vanadyl and molybdenum species in the Keggin structure are the active species for oxidizing MAL to MAA. ${ }^{37}$ Two distinct vanadyl species were detected in the XPS profiles of the supported catalysts, $\mathrm{V}^{5+}$ at $517.1 \mathrm{eV}$ and $\mathrm{V}^{4+}$ at $516.1 \mathrm{eV}^{38}$ (Fig. 6). The ratio of $\mathrm{V}^{4+}$ in $2 \mathrm{HPMV} / \mathrm{CN}-\mathrm{SBA}(45.5 \%)$ was higher than that in HPMV/SBA (33.3\%), with the binding energy of both vanadyl species also being lower for HPMV/SBA (Table S2 $\dagger$ ). These findings suggest that the presence of ammonia decreased the chemical states of the vanadyl species. After calcination, the ratio of $\mathrm{V}^{5+}$ increased from $54.5 \%$ to $64.3 \%$ due to $\mathrm{V}^{4+}$ oxidation by oxygen in the air flow. Following the reaction, the $\mathrm{V}^{5+}$ had decreased to $43.5 \%$ because it was reduced to $\mathrm{V}^{4+}$ by the MAL.

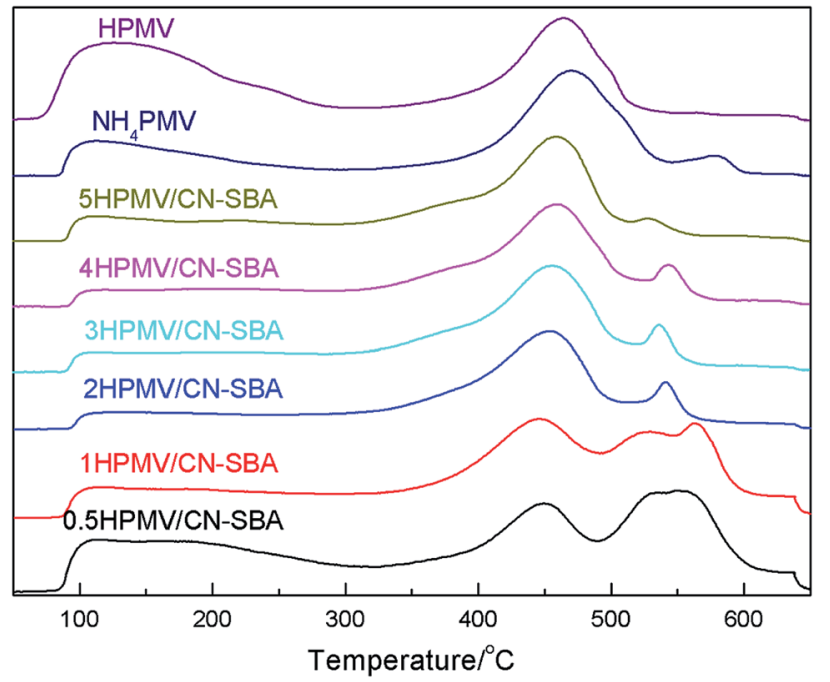

Fig. $6 \mathrm{NH}_{3}$-TPD curves of neat HPMV, neat $\mathrm{NH}_{4} \mathrm{PMV}$, and HPMV/CNSBA after calcination. Catalysts were prepared at $80^{\circ} \mathrm{C}$ with $2 \mathrm{~h}$ mixing and were calcined at $360{ }^{\circ} \mathrm{C}$ for $12 \mathrm{~h}$. 
Two types of molybdenum species with binding energies of 232.8 and $231.6 \mathrm{eV}$ are presented in Fig. S25, $\uparrow$ which could be assigned to $\mathrm{Mo}^{6+}$ and $\mathrm{Mo}^{5+}$, respectively, in the Keggin structure. ${ }^{37}$ The $\mathrm{Mo}^{5+}$ was almost completely oxidized upon calcination, and then reappeared during the reaction. The binding energy of both molybdenum species in 3HPMV/CN-SBA before and after calcination was lower than that for HPMV/SBA. The binding energy change indicates that the ammonia reduced the chemical states of the supported heteropoly compounds, which was also demonstrated in earlier work. ${ }^{39}$ The low chemical states of the vanadyl and molybdenum species are beneficial for improving the selectivity toward MAA.

$\mathbf{N H}_{3}$-TPD. In Fig. 6, two different acid sites were detected in HPMV by NH3-TPD. Weak acid sites appear at $114{ }^{\circ} \mathrm{C}$, and strong acid sites appear at $467{ }^{\circ} \mathrm{C}$. Upon ammonia inclusion, the acid strength of both types of sites changes slightly, while the amount of weak acid sites decreased (Table S4†). The amount of strong acid sites appears to increase with ammonia inclusion. The increase in peak area of the strong acid sites is attributed to the decomposition of the $\mathrm{NH}_{4} \mathrm{PAV}$, which then produces $\mathrm{NH}_{3}$. It should be noted that $1 \mathrm{~g}$ of $\mathrm{NH}_{4} \mathrm{PMV}$ contains $0.56 \mathrm{mmol}$ of ammonia, which is much higher than the amount detected by $\mathrm{NH}_{3}$-TPD, and the actual amount of strong acid sites should decrease with ammonia addition. A new peak emerges at $577{ }^{\circ} \mathrm{C}$ that could potentially be assigned to the ammonia formed from total decomposition of the catalysts. When HPMV was supported on $\mathrm{C}_{3} \mathrm{~N}_{4}$, the strength of the weak acid sites slightly changed, while the acid amount substantially decreased due to the addition of ammonia. Both the strength and amount of strong acid sites of the supported HPA decreased for the same reasons mentioned above. The peaks that emerged between 500-600 ${ }^{\circ} \mathrm{C}$ can potentially be assigned to ammonia formed from the total decomposition of the HPA compounds. With an increase in HPMV loading, the acid strength and amount of weak acid sites slightly changes, while the acid strength and amount of strong acid sites significantly increases. With increasing HPMV loading, increasing numbers protons are available, which can account for the increased acid strength and amount of strong acid sites.

$P$ and Si species in the catalysts. To further confirm the chemical states of SBA-15 and the supported HPA, ${ }^{31} \mathrm{P}$ and ${ }^{29} \mathrm{Si}$ NMR were performed. Fig. 7 shows that only one chemical shift appears at $-5 \mathrm{ppm}$ in the ${ }^{31} \mathrm{P}$ NMR spectrum of HPMV, which can be assigned to $\mathrm{P}$ atoms in Keggin structure. ${ }^{42}$ This suggests that the synthesized HPMV is pure. Fig. 7 shows that one type of phosphorus species was detected at $-4.5 \mathrm{ppm}$ in HPMV/SBA before and after calcination and as well as post-reaction, which can be attributed to the $\mathrm{P}$ in the Keggin structure. The chemical shifts of phosphorus species in HPMV and supported HPMV are different, which may be due to the interaction between HPMV and silica. Following reaction, the chemical shift of $\mathrm{P}$ had moved to $-5.3 \mathrm{ppm}$ due to catalyst reduction by MAL and the removal of absorbed and crystalline water. As detailed in earlier work, a portion of supported HPMV completely decomposes during calcination and reaction, with the released $\mathrm{P}$ volatilized as $\mathrm{P}_{2} \mathrm{O}_{5}$ to the air. ${ }^{37}$ Consequently, no additional $\mathrm{P}$ species are found in the spectra of HPMV/SBA
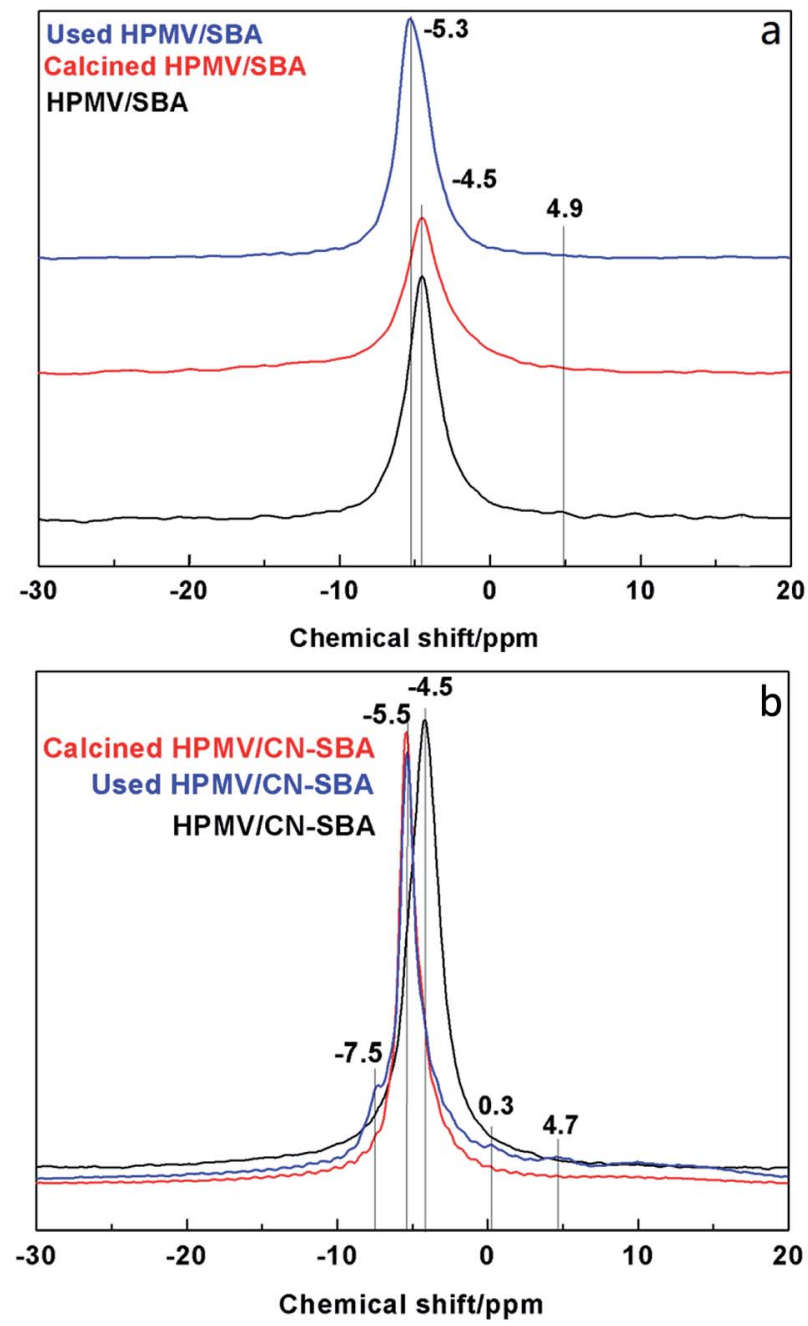

Fig. $7{ }^{31} \mathrm{P}$ NMR spectra of (a) HPMV/SBA and (b) 2HPMV/CN-SBA before and after calcination and reaction.

before and after calcination and reaction. The chemical shift of $\mathrm{P}$ in 3HPMV/CN-SBA was at $-4.5 \mathrm{ppm}$, which was the same as that of HPMV/SBA. After calcination and reaction, the main peak shifted to $-5.5 \mathrm{ppm}$ for the same reason detailed above. An additional peak also emerged at $-7.5 \mathrm{ppm}$, which can be assigned to $\mathrm{P}$ species in a partly decomposed Keggin structure that had formed during the reaction and remained on the surface. ${ }^{37}$ This finding indicates that partially decomposed HPA may exist on the support during reaction, with the presence of the partially decomposed species suggesting that HPMV on $\mathrm{CN}$ SBA is more stable than that on the neat SBA-15.

The HPMV/SBA and HPMV/CN-SBA ${ }^{29} \mathrm{Si}$ NMR spectra are shown in Fig. 8. Chemical shifts at $-110,-102.5$, and -91 ppm can be attributed to the Q4, Q3, and Q2 silicon species in $\mathrm{Si}-(\mathrm{OSi})_{n}(\mathrm{OH})_{4-n}$, respectively. ${ }^{38} \mathrm{Q} n$ is the number of siloxane bonds linking the Si site to the silica framework. The T2 and T3 (Si-R, R is the organic groups) chemical shifts that should appear at -67 and $-54 \mathrm{ppm}$ are almost invisible. For CN-SBA, the chemical shifts of Q4, Q3, and Q2 move to -111, -104 , and $-90 \mathrm{ppm}$, respectively, and appear to arise from the 


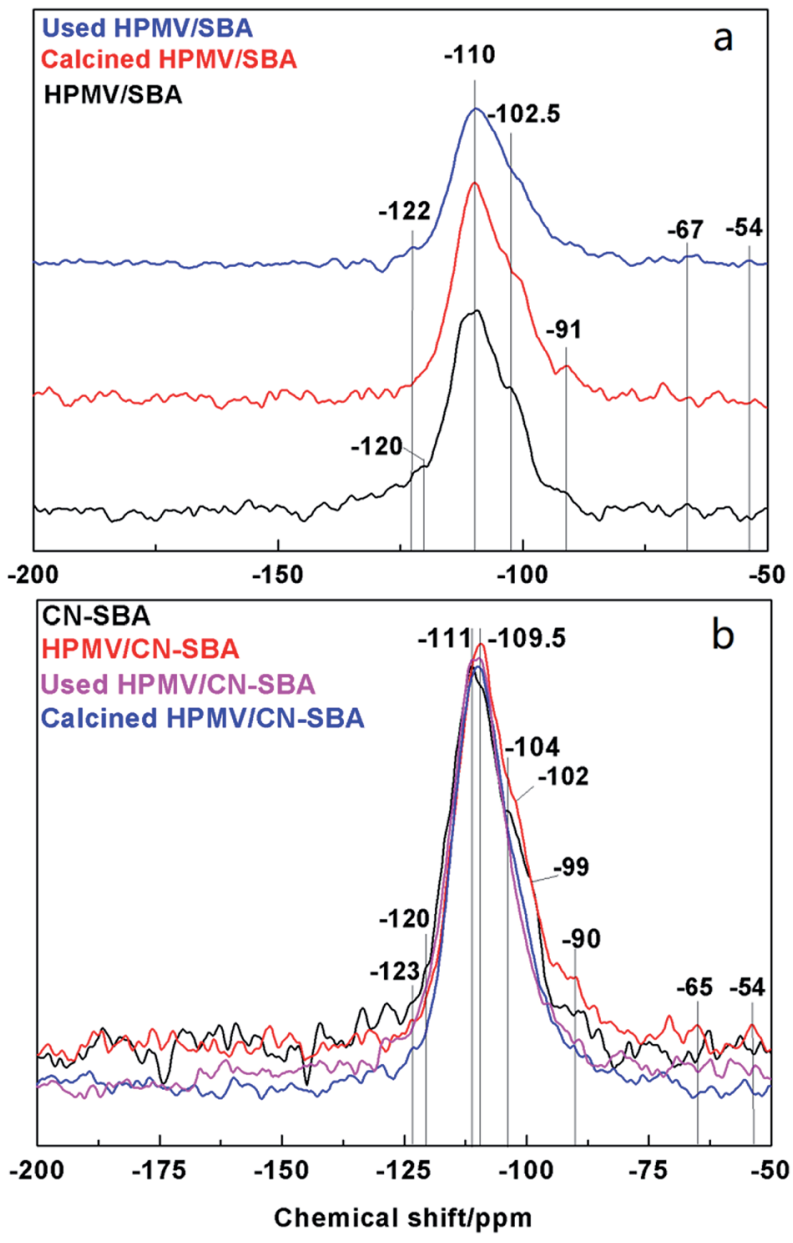

Fig. $8{ }^{29} \mathrm{Si}$ NMR spectra of HPMV/SBA and 2HPMV/CN-SBA before and after calcination and post-reaction.

influence of carbon nitride on the SBA-15 surface. Carbon nitride is rich in electrons that result in silicon species chemically shifting to high field, which also suggests that SBA15 was successfully modified by carbon nitride. A new chemical shift at $-99 \mathrm{ppm}$ can be assigned to silica species interacting with amino groups. When HPMV is added to the CNSBA support, the chemical shift of silicon species in Si$(\mathrm{OSi})_{n}(\mathrm{OH})_{4-n}$ results in movement to -109.5 and $-102 \mathrm{ppm}$, which was the same as that for HPMV/SBA. Disappearance of the chemical shift at $-99 \mathrm{ppm}$ and weakening of the chemical shift at $-90 \mathrm{ppm}$ following calcination may arise from a reaction between HPMV and the amino groups on carbon nitride that causes a decrease in the electron cloud density and breaks the interaction between silica and amino groups. The ${ }^{29} \mathrm{Si}$ NMR results demonstrate that carbon nitride strongly combined with SBA-15, and HPMV remains segregated from SBA-15.

Catalytic performance. Catalyst performance in terms of activity and selectivity are provided in Fig. 9, 10 and S29-S32. $\dagger$ MAL conversion over neat HPMV was the highest at $34.8 \%$ and $290{ }^{\circ} \mathrm{C}$ (Fig. 9). Upon loading HPMV onto the CN-SBA support (0.5HPMV/CN-SBA), MAL conversion was $9.5 \%$ at $290{ }^{\circ} \mathrm{C}$, which

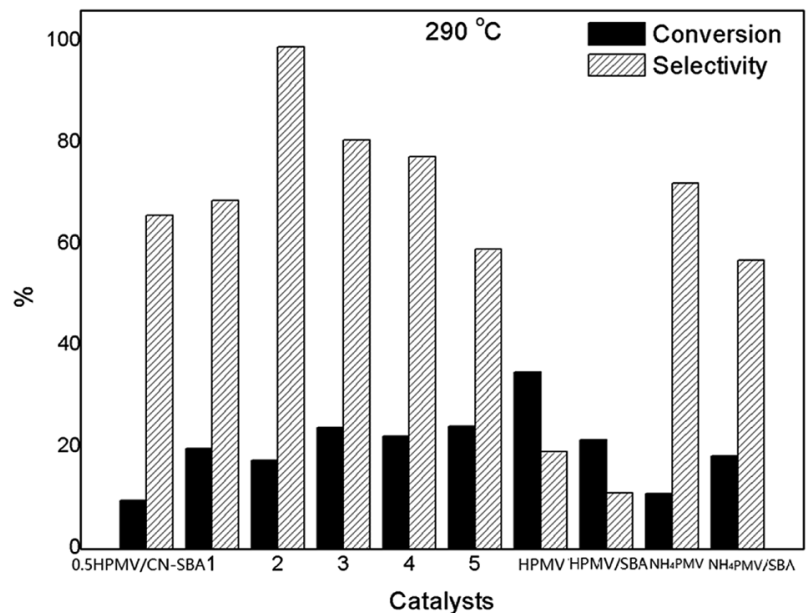

Fig. $9 \mathrm{MAL}$ conversion and MAA selectivity over $0.5 \mathrm{HPMV} / \mathrm{CN}-\mathrm{SBA}$, 1HPMV/CN-SBA (1), 2HPMV/CN-SBA (2), 3HPMV/CN-SBA (3), 4HPMV/ CN-SBA (4), 5HPMV/CN-SBA (5), and HPMV, HPMV/SBA-15, NH $\mathrm{H}_{4} \mathrm{PMV}$, and NHPMV/SBA at $290^{\circ} \mathrm{C}$. (The volume ratios of MAL, $\mathrm{O}_{2}$, and $\mathrm{H}_{2} \mathrm{O}$ in the reactant stream were $4.4 \mathrm{vol} \%, 11.1 \mathrm{vol} \%$, and $17.8 \mathrm{vol} \%$, respectively, with a balance of $\mathrm{N}_{2}$ ).

is much lower than that over HPMV. Increasing HPMV loading from 0.5 to 5 resulted in an increase in MAL conversion to 24.2\%. MAL conversion for the control HPMV/SBA, $\mathrm{NH}_{4} \mathrm{PMV}$, and NHPMV/SBA was $21.4 \%, 10.8 \%$, and $18.3 \%$, respectively. Loading the HPMV onto the CN-SBA support more significantly impacted selectivity toward MAA than activity. The selectivity increased from $19.1 \%$ for the neat HPMV to $65.7 \%$ when loaded onto the support (0.5HPMV/CN-SBA), reaching a maximum of $98.9 \%$ for 2 HPMV/CN-SBA. At HPMV loading beyond 2, the selectivity was diminished. Upon addition of ammonium ion to HPMV, MAA selectivity increased from $19.1 \%$ to $72 \%$ $\left(\mathrm{NH}_{4} \mathrm{PMV}\right)$.

When $\mathrm{NH}_{4} \mathrm{PMV}$ was supported on SBA-15, MAA selectivity decreased to $56.9 \%$. When the reaction was performed at $310{ }^{\circ} \mathrm{C}$, MAL conversion on the supported catalysts increased considerably, while MAA selectivity decreased (Fig. S28†). Additionally, at $310{ }^{\circ} \mathrm{C}$, both MAL conversion and MAA selectivity increased for HPMV and $\mathrm{NH}_{4} \mathrm{PMV}$. Similarly, when the reaction temperature was further increased to $320{ }^{\circ} \mathrm{C}$ (Fig. S30†), MAL conversion on all the catalysts increased, while MAA selectivity on all the catalysts decreased. HPMV was also successfully supported by KIT-6, HY zeolite, $\mathrm{TiO}_{2}$, $\mathrm{Al}_{2} \mathrm{O}_{3}, \mathrm{SiO}_{2}$, CNTs, and $\mathrm{NH}_{3}$-modified CNTs. Although HMPV supported on these supports showed increased catalytic activity, MAA selectivity over HPMV on these supports was much lower than that on CN-SBA (Fig. S29†). The results showed that CN-SBA is the most favourable porous support for HPMV.

Catalyst preparation temperature and mixing time during synthesis were also examined, using $2 \mathrm{HPMV} / \mathrm{CN}-\mathrm{SBA}$ with the conversion and selectivity results at $290{ }^{\circ} \mathrm{C}$ shown in Fig. 10, $\mathrm{S} 29$ and $\mathrm{S} 30 \dagger$. Increasing the preparation temperature (Fig. 10a) provided a small increase in MAL conversion, while MAA selectivity was the most optimal (98.9\%) at a preparation 

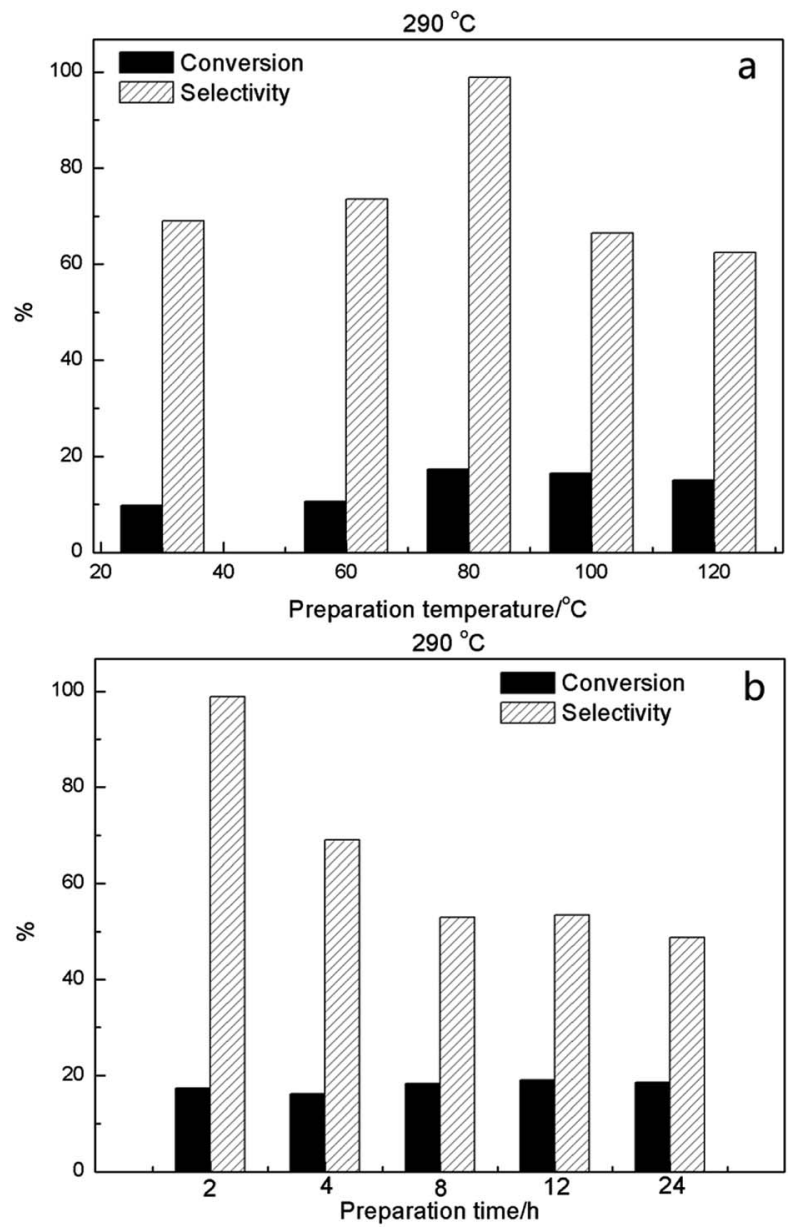

Fig. 10 MAL conversion and MAA selectivity at $290^{\circ} \mathrm{C}$ for $2 \mathrm{HPMV} / \mathrm{CN}-$ SBA prepared at (a) different temperatures and (b) using different synthesis (mixing) times.

temperature of $80{ }^{\circ} \mathrm{C}$. The most suitable mixing time synthesis was found to be $2 \mathrm{~h}$ (Fig. 10b), with longer times resulting in poorer catalyst selectivity, in particular.

\section{Discussion}

Relative to all the control cases, the HPMV/CN-SBA delivered a generally better catalytic performance (Fig. 8), either in terms of conversion or MAA selectivity. Loading HPMV on a silica support resulted in a decrease in both activity and selectivity relative to the neat HPMV. The poorer catalytic performance of HPMV/SBA arose from the decomposition of HPMV into $\mathrm{MoO}_{3}$ and $\mathrm{V}_{2} \mathrm{O}_{5}$, due to interaction between the HPMV and the silica support 17. The poor selectivity is thought to have originated from further MAA oxidation on the $\mathrm{MoO}_{3}$ and $\mathrm{V}_{2} \mathrm{O}_{5}$ to other byproducts. ${ }^{37}$ Including $\mathrm{NH}_{4}{ }^{+}$in the HPMV structure is particularly beneficial for MAA selectivity, although loading it into the SBA-15 mildly counteracted the benefit. FT-IR and XRD results (Fig. S33 and S34 $\dagger$ ) showed that part of the $\mathrm{NH}_{4} \mathrm{PMV}$ on SBA-15 degraded to $\mathrm{MoO}_{3}$ and $\mathrm{V}_{2} \mathrm{O}_{5}$ when supported on the SBA-15. Consequently, it is apparent that it is necessary to separate HPMV from silica to minimize their interaction and avoid partial decomposition of the HPA. For almost all the HPMV catalysts supported on CN-SBA, a comparable or higher MAA selectivity relative to the $\mathrm{NH}_{4} \mathrm{PMV} / \mathrm{SBA}$ was observed. Importantly, no $\mathrm{MoO}_{3}$ or $\mathrm{V}_{2} \mathrm{O}_{5}$ was detected for the HPMV/CN-SBA by any of the characterization techniques, demonstrating that the $\mathrm{CN}$ is crucial for keeping the HPMV from the silica and minimizing HPA decomposition. The CN barrier strategy adopted here is an effective approach for improving and maintaining catalyst performance.

From a MAL conversion perspective, the most optimal performance was exhibited by the neat HPMV because it was able to process the strongest acidity (Fig. S35 and Table S2 $\dagger$ ). The oxidation of MAL to MAA on HPAs follows the Van-Marvel mechanism, whereby strong acidity is positive for MAL adsorption on the catalyst surface, which then promotes higher MAL conversion. ${ }^{43}$ Consequently, MAL conversion on both $\mathrm{NH}_{4} \mathrm{PMV}$ and $\mathrm{NH}_{4} \mathrm{PMV} / \mathrm{SBA}$ was lower than that for HPMV due to their lower acidity levels. When HPMV was loaded onto the CN-SBA, overall, the acidity decreased compared to the neat HPMV, although the effect was moderated with increasing HPMV loading. Interaction of the HPMV with basic amino groups on the CN-SBA surface effectively lessened HPA acidity, and in turn, its capacity for MAL conversion. Additional HPMV acted to partially offset the neutralizing influence of the amino groups, which appeared to reach (and remain at) a maximum HPMV loading of 3. The findings demonstrate that although the surface area of the supported catalysts was considerably larger than that for bulk HPAV (Table 1), any beneficial impact on MAL conversion was overshadowed by the influence of the basic amino groups on the CN-SBA. These findings demonstrate that acidity is the key driver that influences MAL conversion, and while the $\mathrm{CN}$ is important for preserving the HPA structure, its amino groups have a mildly negative impact on catalytic activity.

The presence of ammonium in the catalyst system resulted in a significant increase in selectivity towards MAA. Previous studies showed that ammonia addition decreased the chemical valence of vanadyl and molybdenum species, such that the oxidation susceptibility decreased. This hampered MAL conversion on the CN-SBA-supported HPMV, $\mathrm{NH}_{4} \mathrm{PMV}$, and $\mathrm{NH}_{4}$ PMV/SBA. ${ }^{43}$ However, restricting oxidation susceptibility could also prevent deep oxidation of the MAL and increase MAA selectivity. ${ }^{\mathbf{4 3 4 4}}$ Here, with the increase in the HPMV loading, MAA initially increased to reach a maximum for 2HPMV/CN-SBA, after which it began to decrease. As the HPMV loading increased, the surface area decreased (Table 1), although this was eclipsed by a parallel increase in catalyst acidity (Table $\mathrm{S} 2 \dagger$ ), while the ammonia content remained constant.

The low acidity (e.g., 0.5HPMV/CN-SBA) was not positive for MAL adsorption and MAA desorption, resulting in the observed low MAL conversion and MAA selectivity. However, when acidity was too strong (5HPMV/CN-SBA), deep oxidation of the MAA occurred, which decreased the MAA selectivity while retaining reasonable MAA conversion. Additionally, if the HPMV loading was too low or too high, the decomposition of the HPA to $\mathrm{MoO}_{3}$ or $\mathrm{V}_{2} \mathrm{O}_{5}$ was evident (Fig. S10 $\dagger$ ). Decomposition of HPMV on CNSBA when at a low is envisaged to relate to the small size of 
supported HPA, rendering it unstable at an elevated temperature. In contrast, it is thought that higher HPMV loadings lead to direct interaction between the SBA-15 and the HPA, promoting its partial decomposition. It is suspected that the SBA-15 surface in not entirely covered with $\mathrm{C}_{3} \mathrm{~N}_{4}$. When the HPA is loaded onto CN-SBA, it would preferentially react with and be immobilized by amino groups on the $\mathrm{C}_{3} \mathrm{~N}_{4}$ surface. However, when the $\mathrm{C}_{3} \mathrm{~N}_{4}$ surface area became saturated with HPMA at high loading, excess HPMA was found directly on the silica surface. The ideal HPMV loading appears to be approximately 66.7 wt\% (i.e., 2HPMV/CN-SBA).

Consideration of the catalyst preparation temperature and synthesis (mixing) time showed that they both influenced the performance in terms of MAL conversion and MAA selectivity, with the effect being considerably more pronounced for selectivity. An optimum synthesis temperature of $80^{\circ} \mathrm{C}$ was observed. Catalysts prepared at a temperature below $80{ }^{\circ} \mathrm{C}$ exhibited smaller crystal size, which was detrimental to performance. At synthesis temperatures above $80{ }^{\circ} \mathrm{C}$, FTIR and XRD illustrated the presence of $\mathrm{MoO}_{3}$ in the catalysts (Fig. S8 and $\mathrm{S} 12 \dagger$ ), indicating that partial thermal decomposition of the HPMV had occurred, which was responsible for the diminished performance. In relation to synthesis time, a shorter stirring time ( $2 \mathrm{~h}$, Fig. $10 \mathrm{~b}$ and $\mathrm{S} 32 \dagger)$ delivered more optimal catalyst performance. The XRD spectra again showed an $\mathrm{MoO}_{3}$ presence for synthesis times of $4 \mathrm{~h}$ or more, highlighting a decomposition of the HPMV and explaining the poorer performance.

\section{Experimental}

\section{Catalyst preparation}

HPMV was synthesized according to a previously published procedure described by Kanno et al. ${ }^{11}$ A mixture comprising $5 \mathrm{~g}$ of molybdenum trioxide (Sinopharm Chemical Reagent Co., Ltd.) and $0.287 \mathrm{~g}$ of vanadic anhydride (Tianjin Guangfu Fine Chemical Research Institute) in $100 \mathrm{~mL}$ of deionized water was initially heated to reflux. An $85 \%$ solution of $\mathrm{H}_{3} \mathrm{PO}_{4}$ (Sinopharm Chemical Reagent Co., Ltd.), equal to $0.364 \mathrm{~g}$ of acid, was added dropwise into the turbid liquid. The mixture was vigorously stirred and refluxed for $5 \mathrm{~h}$, and a deep-orange solution was formed. The solid was recovered via filtration, and then, the solution was evaporated at $80{ }^{\circ} \mathrm{C}$ to obtain a bright orange powder. The structure of HPMV was confirmed by FT-IR (Fig. S1†).

SBA-15 was prepared by a process described elsewhere. ${ }^{32}$ In a typical experiment, P123 (4 g, poly(ethylene glycol)-blockpoly(propylene glycol)-block-poly(ethylene glycol), Aldrich Chemical Co.) was dissolved in $125 \mathrm{~mL}$ of $1.8 \mathrm{wt} \%$ hydrochloric acid (Sinopharm Chemical Reagent Co. Ltd.) solution at $40{ }^{\circ} \mathrm{C}$. Tetraethoxysilane (8.16 g, TEOS, Sinopharm Chemical Reagent Co. Ltd.) was then added dropwise into the solution, which was hydrolyzed for $24 \mathrm{~h}$ at $40{ }^{\circ} \mathrm{C}$. Following hydrolysis, the mixture was hydrothermally treated at $100{ }^{\circ} \mathrm{C}$ for $24 \mathrm{~h}$. The resulting solid was recovered using filtration, washed with water and alcohol, and calcined at $550{ }^{\circ} \mathrm{C}$ for $6 \mathrm{~h}$. It was characterized by small angle XRD (Fig. S2†).
Carbon nitride (CN)-modified SBA-15 (CN-SBA) was prepared by dissolving $0.8 \mathrm{~g}$ of melamine into $30 \mathrm{~mL}$ of deionized water at $80{ }^{\circ} \mathrm{C}$. After the melamine was completely dissolved, $2 \mathrm{~g}$ of SBA-15 was added to the solution with the mixture vigorously stirring, and the stirring continued for $2 \mathrm{~h}$. Water was then evaporated from the mixture at $110^{\circ} \mathrm{C}$, and the solid dried in an oven at $80{ }^{\circ} \mathrm{C}$ for $12 \mathrm{~h}$. The dried solid was calcined in an airtight magnetic boat at a ramp rate of $3{ }^{\circ} \mathrm{C} \min ^{-1}$ to $550{ }^{\circ} \mathrm{C}$, where the temperature was held for $3 \mathrm{~h}$. The amino group content of CN-SBA was $2.2 \mathrm{mmol} \mathrm{g}^{-1}$, which was evaluated using $\mathrm{CO}_{2}$-TPD and energy dispersive X-ray spectroscopy (EDS) (Fig. S3 and S4†).

HPMV was loaded onto the CN-SBA using the following procedure. First, $2 \mathrm{~g}$ of HPMV was dissolved in $10 \mathrm{~mL}$ of deionized water and added dropwise to $1 \mathrm{~g}$ of CN-SBA powder. The mixture was then stirred at $80^{\circ} \mathrm{C}$ for $2 \mathrm{~h}$. The remaining water was evaporated, and the solid dried in an oven at $80^{\circ} \mathrm{C}$ for $12 \mathrm{~h}$. Catalyst nomenclature is in the form of $n \mathrm{HPMV} / \mathrm{CN}-\mathrm{SBA}$, where $n$ denotes the HPMV loading ( $n=$ (weight of HPMV, g)/(weight of CN-SBA, g)). HPMV was also loaded onto neat SBA-15 ( $n$ HPMV/ SBA) following the same procedure described above.

As a control to evaluate the HPMV/CN-SBA, $\mathrm{NH}_{4} \mathrm{H}_{3} \mathrm{PMo}_{11^{-}}$ $\mathrm{VO}_{40}(\mathrm{NHPMV})$ was synthesized by mixing $2 \mathrm{~g}$ of HPMV in $10 \mathrm{~mL}$ of deionized water with $0.06 \mathrm{~g}$ of $\mathrm{NH}_{4} \mathrm{Cl}$ (Sinopharm Chemical Reagent Co., Ltd.) in $10 \mathrm{~mL}$ of deionized water and stirring at $80{ }^{\circ} \mathrm{C}$ for $2 \mathrm{~h}$. The remaining water was evaporated, and the resulting yellow solid was dried in an oven at $80{ }^{\circ} \mathrm{C}$ for $12 \mathrm{~h}$.

NHPMV supported on SBA-15 (NHPMV/SBA) was prepared by dissolving $0.06 \mathrm{~g} \mathrm{NH}_{4} \mathrm{Cl}$ in $10 \mathrm{~mL}$ deionized water, and the solution was added dropwise onto $3 \mathrm{~g}$ of dried HPMV/SBA. The mixture was then stirred at $80{ }^{\circ} \mathrm{C}$ for $2 \mathrm{~h}$. Any remaining water was evaporated, and the recovered yellow solid was dried in an oven at $80{ }^{\circ} \mathrm{C}$ for $12 \mathrm{~h}$.

Following synthesis, all catalysts were calcined at $360{ }^{\circ} \mathrm{C}$ in air at a flow rate of $80 \mathrm{~mL} \mathrm{~min}^{-1}$ in a pipe furnace (SK-G03123K, Zhonghuan Co., Ltd., Tianjin).

\section{Catalyst characterization}

Fourier transform infrared (FT-IR) spectrograms of the catalysts were recorded from 4000 to $400 \mathrm{~cm}^{-1}$ on a Nicolet 380 FT-IR spectrometer (Thermal Electron Corporation) with anhydrous $\mathrm{KBr}$ as the standard. The crystal phase of the catalysts was evaluated by X-ray diffraction (XRD, Bruker D8 Advance X-ray powder diffractometer) with $\mathrm{Cu} \mathrm{Ka}$ radiation $(\lambda=0.154 \mathrm{~nm})$ as the X-ray source. Thermal stability of the samples was analyzed using a thermogravimetric differential thermal analyzer (TG/DTA, DTG-60H, Shimadzu, Japan). Approximately $10 \mathrm{mg}$ of the sample was heated from 25 to $700{ }^{\circ} \mathrm{C}$ at $5{ }^{\circ} \mathrm{C} \mathrm{min}{ }^{-1}$ in an air atmosphere (flow rate $10 \mathrm{~mL} \mathrm{~min}^{-1}$ ). Catalyst acidity was determined using $\mathrm{NH}_{3}$ temperature-programmed desorption analysis on a Micromeritics AutoChem II Chemisorption Analyzer. $\mathrm{NH}_{3}$ desorption was recorded over the temperature range of 50 to $650{ }^{\circ} \mathrm{C}$ at a ramp rate of $10{ }^{\circ} \mathrm{C} \mathrm{min}{ }^{-1}$. Catalyst morphology was observed using scanning electron microscopy (SEM, SU8020, Hitachi, Japan). Oxidation states of the catalyst components were evaluated by X-ray photoelectron spectroscopy (XPS, Kratos Axis Ultra DLD spectrometer) with 
monochromatic $\mathrm{Al} \mathrm{K} \alpha$ radiation as the source. Surface area and pore structure of the catalysts were analyzed using $\mathrm{N}_{2}$ physisorption on a Micromeritics ASAP 2460 (US) via the BET and Barrett-Joyner-Halenda (BJH) methods, respectively. Prior to analysis, the samples were pretreated by heating at $200{ }^{\circ} \mathrm{C}$ for $4 \mathrm{~h}$ under vacuum. Silicon and phosphorus species in the catalysts were detected by ${ }^{29} \mathrm{Si}$ NMR and ${ }^{31} \mathrm{P}$ NMR [Bruker Avance III (400M)] analyses at $12 \mathrm{kHz}$, respectively.

\section{Catalyst performance}

Catalyst performance was evaluated at atmospheric pressure in a $400 \mathrm{~mm}$-long fixed bed reactor with an internal diameter of $6 \mathrm{~mm}$. Prior to performance assessment, the bulk catalysts were crushed and screened to a mesh size fraction of 20 to 40 . Next, $0.8 \mathrm{~g}$ of the catalyst was loaded into the reactor. Air was fed through the catalyst bed at a rate of $10 \mathrm{~mL} \mathrm{~min}^{-1}$, and the bed temperature was increased to $310{ }^{\circ} \mathrm{C}$. The reactant gas stream, comprising $\mathrm{N}_{2}$ that had passed through a bubbler containing MAL at $19{ }^{\circ} \mathrm{C}$ with water added via an advection pump, was passed through the catalyst bed at $19 \mathrm{~mL} \mathrm{~min}^{-1}$. The volume percent (vol\%) of MAL, $\mathrm{O}_{2}$, and $\mathrm{H}_{2} \mathrm{O}$ in the reactant stream was $4.4,11.1$, and 17.8 , respectively, with the balance composed of $\mathrm{N}_{2}$. Once the reaction had proceeded for $1 \mathrm{~h}$, the products were analyzed by a gas chromatograph (7890B, Agilent Technologies Co. Ltd.) with a flame ionization detector and a DB-FFAP capillary column. The column tank temperature increased from 40 to $240{ }^{\circ} \mathrm{C}$ at a rate of $25{ }^{\circ} \mathrm{C} \mathrm{min}{ }^{-1}$ and was maintained at $240{ }^{\circ} \mathrm{C}$ for $5 \mathrm{~min}$; the injection temperature was $180^{\circ} \mathrm{C}$. The MAA selectivity was calculated by the molar amount of MAA being divided by the converted MAL molar amount (Scheme 1).

MAL conversion was calculated by eqn (1), and selectivity toward MAA was calculated by eqn (2).

$$
\text { MAL conversion }=(1-F(\mathrm{MAL}) \text { tail }) / F(\mathrm{MAL})
$$

MAA selectivity $=F(\mathrm{MAA}) /[F(\mathrm{MAL}) \times$ Conversion $]$

where $F(\mathrm{MAL})$ denotes the initial MAL molar flow rate $\left(\mathrm{mol} \mathrm{min}^{-1}\right)$, and $F(\mathrm{MAL})$ tail denotes the MAL molar flow rate after reaction.

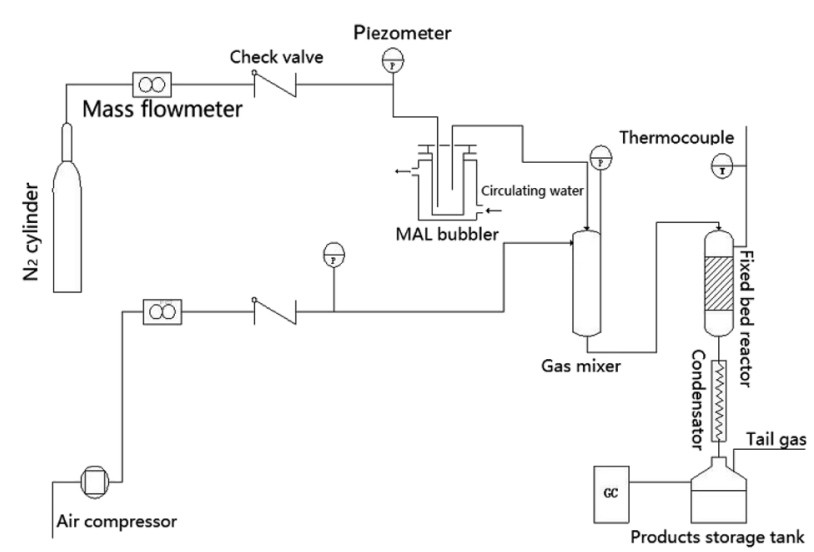

Scheme 1 Catalytic process for oxidation of MAL to MAA.

\section{Conclusions}

In this work, modified SBA-15 was used as a support for HPMV for the oxidation of MAL to MAA. The CN modification offered twin benefits in terms of improving catalyst performance: (i) it acted as a barrier to restrict direct interaction between HPMV and SBA-15, improving HPMV stability; (ii) it furnished the catalyst support with amino groups, which were advantageous for MAA selectivity. In the absence of $\mathrm{C}_{3} \mathrm{~N}_{4}$, HPMV was partially decomposed to $\mathrm{MoO}_{3}$ and $\mathrm{V}_{2} \mathrm{O}_{5}$ by SBA-15, which was detrimental to performance. At an optimum HPMV catalyst formulation of 2HPMV/CN-SBA, the MAA selectivity was improved by more than five times over neat HPMV. Lower and higher HMPV loadings resulted in partial decomposition of the HPMV, which was attributed to smaller less thermally stable HPMV deposits and excess HPMV spillover onto the SBA-15, respectively. Synthesis temperature and mixing time were found to be important for catalyst performance, with the optimized conditions identified to be $80^{\circ} \mathrm{C}$ and $2 \mathrm{~h}$ stirring, respectively. These findings reveal a new approach that overcomes selectivity and stability challenges associated with using supported heteropoly acids as catalysts for oxidation reactions.

\section{Conflicts of interest}

There are no conflicts to declare.

\section{Acknowledgements}

The authors gratefully acknowledge financial support from the Youth Foundation of the Education Department of Hebei Province (No. QN2019230) and the Natural Science Foundation of Hebei Province Youth Project (No. B2019208333).

\section{Notes and references}

$1 \mathrm{~J}$. W. Bauer, Ultmann's Encyclopedia of Industrial Chemistry, Wiley-VCH, Weinheim, 2002.

2 K. Nagai, Appl. Catal., A, 2001, 221, 367-377.

3 N. Mizuno and M. Misono, Chem. Rev., 1998, 98, 199-217.

4 M. Misono, ChemComm, 2001, 1141-1152.

5 L. M. G. Sainero, S. Damyanova and J. L. G. Fierro, Appl. Catal., A, 2001, 208, 63-75.

6 N. K. Kala Raj, S. S. Deshpande, R. H. Ingle, T. Raja and P. Manikandan, Catal. Lett., 2004, 98, 217-224.

7 A. Popa, V. Sasca and J. Halasz, Appl. Surf. Sci., 2008, 255, 1830-1835.

8 A. A. Spojakina, N. G. Kostova, B. Sow, M. W. Stamenova and K. Jiratova, Catal. Today, 2001, 65315-65321.

9 N. Legagneux, J. M. Basset, A. Thomas, F. Lefebvre, A. Goguet, J. Sab and C. Hardacre, Dalton Trans., 2009, 2235-2240.

10 N. Ballarini, F. Candiracci, F. Cavani, H. Degrand, J. L. Dubois, G. Lucarelli, M. Margotti, A. Patinet, A. Pigamo and F. Trifirò, Appl. Catal., A, 2007, 325, 263-269.

11 M. Kanno, T. Yasukawa, W. Ninomiya, K. Ooyachi and Y. Kamiya, J. Catal., 2010, 273, 1-8. 
12 M. Kanno, Y. Miura, T. Yasukawa, T. Hasegawa, W. Ninomiya, K. Ooyachi, H. Imai, T. Tatsumi and Y. Kamiya, Catal. Commun., 2011, 13, 59-62.

13 M. Hino and K. Arata, Green Chem., 2001, 3, 170-172.

14 J. M. Tatibout, C. Montalescot and K. Brückman, Appl. Catal., A, 1996, 138, 11-16.

15 Y. Guo, K. Li, X. Yu and J. H. Clark, Appl. Catal., B, 2008, 81, 182-191.

16 A. Gaurav, F. T. T. Ng and G. L. Rempel, Green Energy \& Environment, 2016, 1, 62-74.

17 L. Zhou, L. Wang, H. Wang, Y. Cao, R. Yan and S. Zhang, J. Thermodyn. Catal., 2016, 7(4), 176-181.

18 A. Y. Liu and M. L. Cohen, Science, 1989, 245, 841-842.

19 M. Kawaguchi, S. Yagi and H. Enomoto, Carbon, 2004, 423, 45-350.

20 Y. Qiu and L. Gao, Chem. Commun., 2003, 9, 2378-2379.

21 Q. Guo, Q. Yang, L. Zhu, C. Yi, S. Zhang and Y. Xie, Solid State Commun., 2004, 132, 369-374.

22 E. Kroke and M. Schwarz, Coord. Chem. Rev., 2004, 248, 493532.

23 Y. J. Bai, B. Lu, Z. G. Liu, L. Li, D. L. Cui, X. G. Xu and Q. L. Wang, J. Cryst. Growth, 2003, 247, 505-508.

24 J. L. Zimmerman, R. Williams, V. N. Khabashesku and J. L. Margrave, Nano Lett., 2001, 1731-1734.

25 V. N. Khabashesku, J. L. Zimmerman and J. L. Margrave, Chem. Mater., 2000, 12, 3264-3270.

26 J. Wang, D. R. Miller and E. G. Gillan, Carbon, 2003, 41, 2031-2037.

27 D. R. Miller, J. Wang and E. G. Gillan, J. Mater. Chem., 2002, 12, 2463-2469.

28 M. Kim, S. Hwang and J. S. Yu, J. Mater. Chem., 2007, 17, 1656-1659.
29 J. Kouvetakis, A. Bondari, M. Todd, B. Wilkens and N. Cave, Chem. Mater., 1994, 6, 811-814.

30 X. Chen, Y. S. Jun, K. Takanabe, K. Maeda, K. Domen, X. Fu, M. Antonietti and X. Wang, Chem. Mater., 2009, 21, 40934095.

31 D. Zhao, J. Feng, Q. Huo, N. Melosh, N. Fredrickson, B. F. Chmelka and G. D. Stucky, Science, 1998, 279, 548-552.

32 L. Marosi and C. O. Areán, J. Catal., 2003, 213, 235-240.

33 D. Zhao, Q. Huo, J. Feng, B. F. Chmelka and G. D. Stucky, J. Am. Chem. Soc., 1998, 120, 6024-6036.

34 H. Zhang, R. Yan, L. Yang, Y. Diao, L. Wang and S. Zhang, Ind. Eng. Chem. Res., 2013, 52, 4484-4490.

35 B. Lei, B. Li, H. Zhang, L. Zhang and W. Li, J. Phys. Chem. C, 2007, 111(30), 11291-11301.

36 X. Fan, L. Zhang, R. Cheng, M. Wang, M. Li, Y. Zhou and J. Shi, ACS Catal., 2015, 5(9), 5008-5015.

37 L. Zhou, L. Wang, S. Zhang, R. Yan and Y. Diao, J. Catal., 2015, 329, 431-440.

38 K. Mukhopadhyay, A. Ghoshb and R. Kumar, Chem. Commun., 2002, 2404-2405.

39 Y. L. Cao, L. Wang, L. L. Zhou, G. J. Zhang, B. H. Xu and S. J. Zhang, Ind. Eng. Chem. Res., 2017, 56(3), 653-664.

40 F. Jing, B. Katryniok, F. Dumeignil, E. Bordes-Richard and S. Paul, J. Catal., 2014, 309, 121-135.

41 I. V. Kozhevnikov, J. Mol. Catal. A: Chem., 2009, 305, 104-111. 42 S. Ganapathy, M. Fournier, J. F. Paul, L. Delevoye, M. Guelton and J. P. Amoureux, J. Am. Chem. Soc., 2002, 124, 7821-7828.

43 Y. L. Cao, L. Wang, L. L. Zhou, B. H. Xu, Y. Y. Diao and S. J. Zhang, J. Ind. Eng. Chem., 2018, 65, 254-263.

44 L. Zhou, L. Wang, Y. Diao, R. Yan and S. Zhang, Mol. Catal., 2017, 433, 153-161. 